\title{
All-Fiber Pulsed Lasers Passively Mode-Locked by Transferable Vertically Aligned Carbon Nanotube Film
}

\author{
Yong-Won Song, Shinji Yamashita \\ Department of Electronic Engineering, University of Tokyo, Tokyo 113-8656, Japan \\ Erik Einarsson, Shigeo Maruyama \\ Department of Mechanical Engineering, University of Tokyo, Tokyo 113-8656, Japan
}

An all-fiber passive laser mode locking is realized with a vertically aligned single-walled carbon nanotube film that can be transferred onto an arbitrary substrate using only hot water. A D-shaped fiber is employed as the substrate for the evanescent filed interaction of propagating light with the nanotubes. The scheme highlights the efficient interaction achieved by the nanotube alignment as well as dramatically simplified device preparation process. The demonstrated pulsed laser output has $2.9 \mathrm{~nm}$ of spectral full width at half maximum, and a 20.8 MHz repetition rate. @2007 Optical Society of America

OCIS codes: $140.4050,320.5550,190.4400$ 
Passively mode-locked lasers have been spotlighted during past decades due to their advantages over the active schemes including ultrashort pulse formation as well as the simple and robust laser structure. ${ }^{1,2}$ Additional researches on the passive mode-locking have been motivated by introducing carbon nanotubes (CNTs), the innovative nonlinear optical materials that provide saturable absorption functionality with notable attractions including wide operating bandwidth ranging from 1 to $2 \mu \mathrm{m}$, ultra-short recovery time ( 500 fs), high optical damage threshold, and excellent compatibility with fibers and nano-devices due to their extremely miniaturized foot print. ${ }^{3}$ Given the elucidated works on the morphology control of the individual CNTs, ${ }^{4,5}$ the researches on CNT-based devices are focused on the diversification of the CNT mode-locked lasers in terms of operating wavelength, repetition rate, novel mode-locking scheme, and matrix material for CNT dispersion. ${ }^{6-9}$ Recently, we propose and demonstrate a novel all-fiber modelocking scheme in which the evanescent field of the propagating light in the fiber interacts with the CNTs to induce loss modulation in the laser cavity. ${ }^{10,11}$ In the early stages of the demonstration, CNTs are sprayed randomly onto either a D-shaped fiber or tapered fiber that can broaden the propagating mode, thereby the mode can "feel" the outside of the optical fiber in order to interact with the deposited materials. In spite of the big-hop given by the lateral interaction schemes, the researches still suffer from the complex and difficult preparation process for CNT mode-lockers as well as the limited interaction of the randomized CNTs with the polarized beam.

In this work, we introduce a new scheme for the CNT mode-locker incorporating vertically aligned single-walled carbon nanotube (VA-SWNT) film that can be attached onto an arbitrary substrate using only hot water. ${ }^{12}$ This new scheme highlights some remarkable advantages including (i) simple and safe CNT deposition process, (ii) maximized CNT 
interaction with the field of propagating light due to the CNT alignment, therefore low CNT density threshold to achieve the mode-locking, and (iii) high ( 100\%) yield rate and reliability for the device manufacturing. For the all-fiber configuration, the VA-SWNT film is attached onto a D-shaped fiber.

It is well known that the CNT has outstanding nonlinear optical properties such as saturable absorption. Importantly, it is shown theoretically and experimentally that the absorption of the CNT can be maximized when the field of the incident beam is parallel with the CNT axis. This anisotropic optical absorption property of the CNT is considered to be from $\pi$ plasmon excitation. ${ }^{13,14}$ Fig. 1(a) schematically illustrates that only the $\mathrm{LP}_{01}-\mathrm{Y}$ mode can be absorbed by the CNT that is aligned along the Y-direction when the light propagates along the Zdirection. In our conventional attempts, ${ }^{10}$ the CNTs were deposited 2-dimensionally (see Fig. 1(b)) onto the substrate with randomized directions, therefore, when the beam propagates with a wave vector $\mathbf{k}_{\mathbf{z}}$, the horizontally polarized beam ( $\mathrm{LP}_{01}-\mathrm{X}$ mode) interacts with only a part of the CNTs. On the other hand, as can be seen in Fig. 1(c), the $\mathrm{LP}_{01}-\mathrm{Y}$ mode of the beam with $\mathbf{k}_{\mathbf{z}}$ is broadened wider than the $\mathrm{LP}_{01}-\mathrm{X}$ mode in the $\mathrm{D}$-shaped fiber, thereby ensuring higher intensity of the optical field at the surface of the optical fiber can interact with almost all of the VASWNTs maximizing the nonlinear effects.

The VA-SWNT films prepared by the alcohol catalytic chemical vapor deposition (ACCVD) method are optimized at $\sim 800^{\circ} \mathrm{C}$ with a deposition time of $\sim 10$ minutes. ${ }^{12}$ Catalyst particles are loaded onto quartz substrates by dip-coating into an ethanol solution incorporating Mo-Co bimetallic acetates, resulting in finely monodispersed and stabilized catalyst deposition on the quartz surface. For the SWNT growth, the catalyst-coated substrate is inserted into a tube furnace and heated under $40 \mathrm{kPa}$ of $\mathrm{Ar} / \mathrm{H}_{2}$ gas $\left(3 \% \mathrm{H}_{2}\right)$. Ethanol is supplied as a carbon source, 
forming into SWNTs on the surface of the metal catalyst particles. As the CNTs grow the high nanotube density causes the initially randomized growth direction to align perpendicular to the substrate, resulting in vertically aligned growth. Fig. 2(a) is a SEM image revealing the morphology of the as-grown VA-SWNT film on quartz substrates. The aligned CNT bundles can be identified in the image. Fig. 2(b) shows a carpet-like film that is peeled from the substrate. The optical absorption properties of the film remain the same before and after the film transfer. Fig. 3 depicts the absorption spectra of the films measured with the films at an angle of $45^{\circ}$ with respect to the incident beam. Considering that the absorbance of the CNT is proportional to $\sin ^{2} \theta$ (where $\theta$ is the angle between the CNT and the incident beam), ${ }^{5,15}$ we conclude that the actual nonlinear absorption of the sample grown at $800^{\circ} \mathrm{C}$ is higher than $5 \%$ at $1550 \mathrm{~nm}$. In this work, in order to realize an all-fiber mode-locker, the film is peeled by submersion into hot water $\left(60^{\circ} \mathrm{C}\right)$ from the quartz surface, and then attached onto the flat face of a D-shaped fiber. The Dshaped fiber is prepared by polishing a single mode fiber (SMF). During the D-shaping, the insertion loss is checked in the water, and the final loss is set to $\sim 5 \mathrm{~dB}$. The VA-SWNT film ensures more than 2-dB improvement of intracavity optical power for the mode locking operation compared with our previous work using the CNT spray method. After adding the film on the D-shaped fiber, the device is dried for 2 hours at $80^{\circ} \mathrm{C}$. Our new preparation scheme can guarantee almost $100 \%$ yield by the safe and easy process to form CNT devices. Consequently, we realize a more efficient laser mode-locker with the long, lateral SWNT-light interaction length ( $\sim 5 \mathrm{~mm})$ as well as the strong light absorption by the aligned SWNTs.

The demonstrated laser has a ring cavity structure (see Fig. 4) with an Erbium-doped fiber amplifier (EDFA) as a gain medium. In order to minimize the deleterious reflection to the CNT mode-locker isolators are inserted after the mode-locker and at the output port. Polarization 
controller (PC) optimizes the round-trip polarization in the laser cavity. An extra SMF is added to adjust the intra-cavity dispersion. $5 \%$ of the intracavity lasing light is tapped for the laser output while the other $95 \%$ of the power is fed back into the laser cavity. When the EDFA is pumped with $400 \mathrm{~mA}$, the mode-locking starts and shows a stable operation. In order to achieve better pulse quality, higher gain is introduced into the cavity. Fig. 5(a) shows the output spectrum of the laser measured with $0.1 \mathrm{~nm}$ resolution. The center wavelength is $1562.8 \mathrm{~nm}$, and the $3 \mathrm{~dB}$ spectral width is $2.9 \mathrm{~nm}$, indicating the temporal pulse of $883 \mathrm{fs}$ when a transform-limited sech ${ }^{2}$ pulse waveform is assumed. Fig. 5(b) shows the measured autocorrelation trace of the pulsed output indicating that the temporal pulse width of $850 \mathrm{fs}$ approaches the calculated value. As can be seen in Fig. 5(c), the output pulse train verifies the pulse formation by our VA-SWNT film mode locker. The repetition rate of the laser is $20.8 \mathrm{MHz}$, and the average output power is 12.0 $\mathrm{dBm}$.

As a result, the functionality of our simple and efficient scheme is verified successfully by producing sub-picosecond pulsed laser output. We expect to explore further applications of our controlled SWNT film in the fields of ultra-fast optical switches, polarization management as well as controlled field emissions. 


\section{References}

1 H. A. Haus, IEEE J. Select. Top. Quantum Electron. 6, 1173 (2000).

2 L. Krainer, R. Paschotta, M. Moser, U. Keller, App. Phys. Lett. 77, 2104 (2000).

3 S. Y. Set, H. Yaguchi, Y. Tanaka, M. Jablonski, IEEE J. Select. Top. Quantum Electron. 10, 137 (2004).

4 S. Yamashita, Y. Inoue, S. Maruyama, Y. Murakami, H. Yaguchi, M. Jablonski, S. Y. Set, Opt. Lett. 29, 1581 (2004).

5 S. Yoo, Y. Jung, D. Lee, W. Han, K. Oh, Y. Murakami, T. Edamura, S. Maruyama, Opt. Lett. 30, 3201 (2005).

6 S. Yamashita, Y. Inoue, H. Yaguchi, M. Jablonski, S. Y. Set, European Conference on Optical Communication (ECOC), paper Th.1.3.4. (2004)

7 S. Yamashita, Y. Inoue, K. Hsu, T. Kotake, H. Yaguchi, D. Tanaka, M. Joblonski, S. Y. Set, IEEE Photon. Technol. Lett. 17, 750 (2005).

8 S. Tatsuura, M. Furuki, Y. Sato, I. Iwasa, M. Tian, H. Mitsu, Adv. Mater. 15, 534 (2003).

9 A. G. Rozhin, Y. Sakakibara, S. Namiki, M. Tokumoto, H. Kataura, App. Phys. Lett. 88, 051118 (2006).

10 Y. W. Song, S. Yamashita, C. S. Goh, S. Y. Set, Opt. Lett. 32, 148 (2007).

11 Y. W. Song, K. Morimune, S. Y. Set, S. Yamashita, App. Phys. Lett. 90, 021101 (2007).

12 Y. Murakami, S. Maruyama, Chem. Phys. Lett. 422, 575 (2006).

13 Y. Murakami, E. Einarsson, T. Edamura, S. Maruyama, Phys. Rev. Lett. 94, 087402 (2005).

14 B. W. Reed, M. Sarikaya, Phys. Rev. B 64, 195404 (2001).

15 Y. Murakami, E. Einarsson, T. Edamura, S. Maruyama, Carbon 43, 2664 (2005). 


\section{List of Figure Captions}

Figure 1 (a) Schematic explanation of anisotropic optical absorption of an individual CNT. (b) Conventional operating scheme: Partial absorption of the X-polarized mode by randomized CNTs sprayed onto the X-Z plane. (c) New proposed scheme: Maximized absorption of the Ypolarized mode by the vertically aligned SWNT film.

Figure 2 (a) SEM image of the VA-SWNT film grown on a quartz substrate $(\times 2 \mathrm{k})$. (b) Carpetlike VA-SWNT film peeled away from the substrate $(\times 3 k)$.

Figure 3 Absorption spectra of the SWNT films measured at $45^{\circ}$ with respect to the incident beam.

Figure 4 Demonstrated pulsed laser setup using a VA-SWNT film.

Figure 5 (a) Output spectrum of our pulsed laser with FWHM of 2.9 nm. (b) Autocorrelation trace of the pulse showing the width of $850 \mathrm{fs}$. (c) Verification of the pulsed output with pulse train. The repetition rate is $20.8 \mathrm{MHz}$. 
Figures

\section{Figure 1}

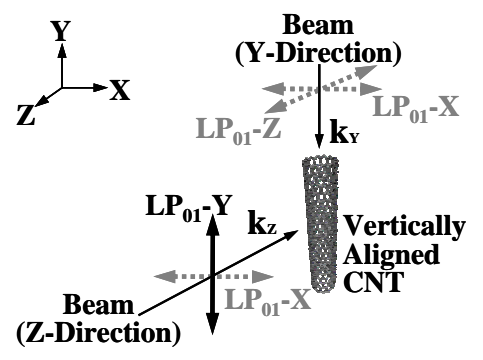

(a)

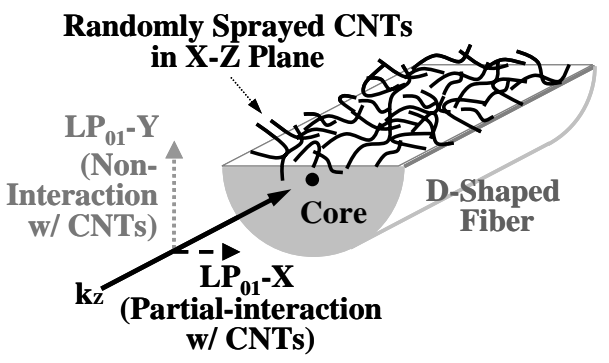

(b)

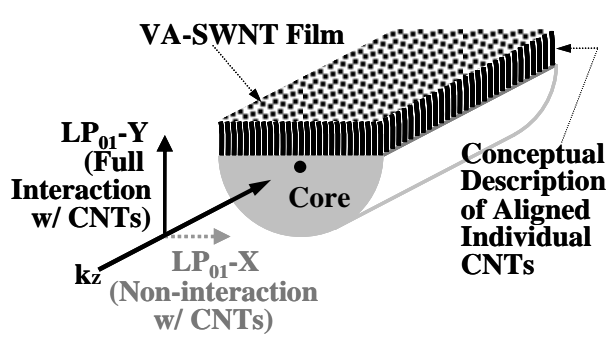

(c) 
Figure 2

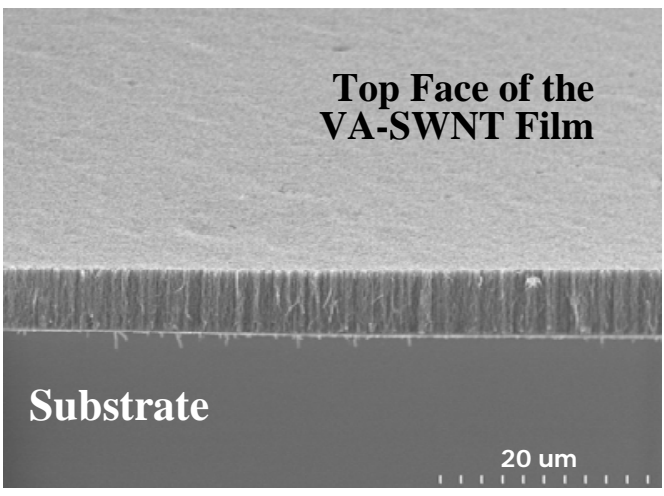

(a)

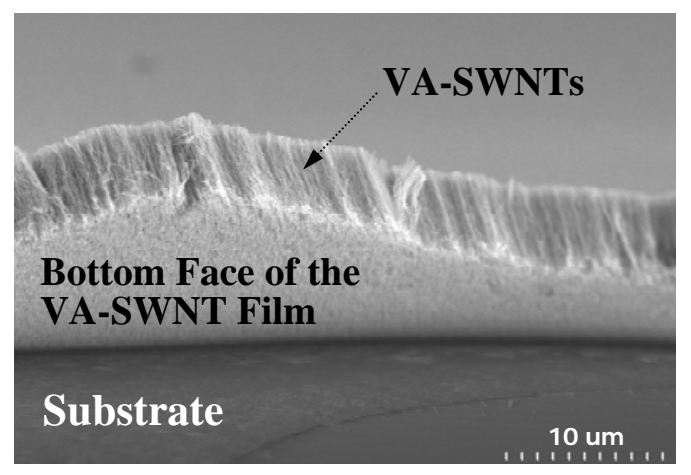

(b) 
Figure 3

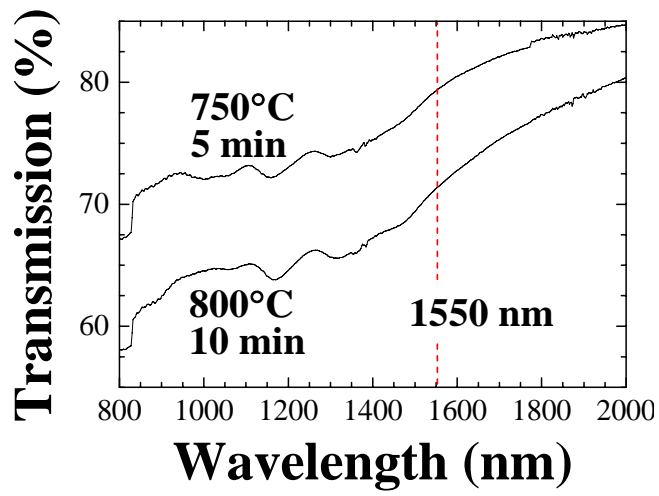




\section{Figure 4}

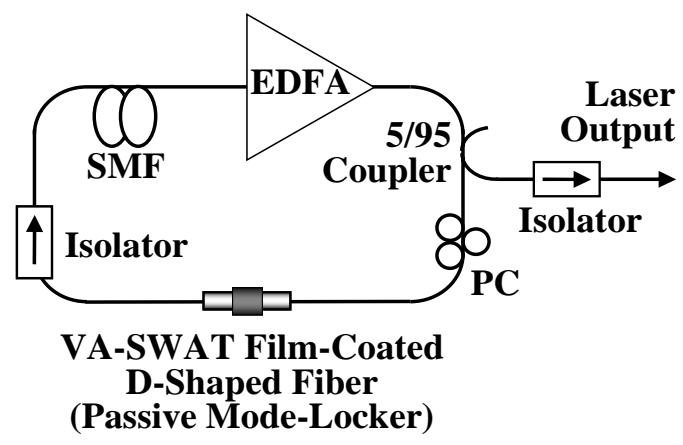




\section{Figure 5}

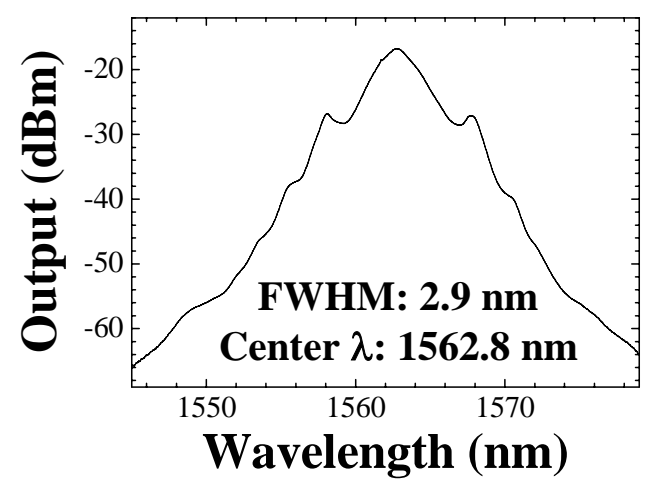

(a)

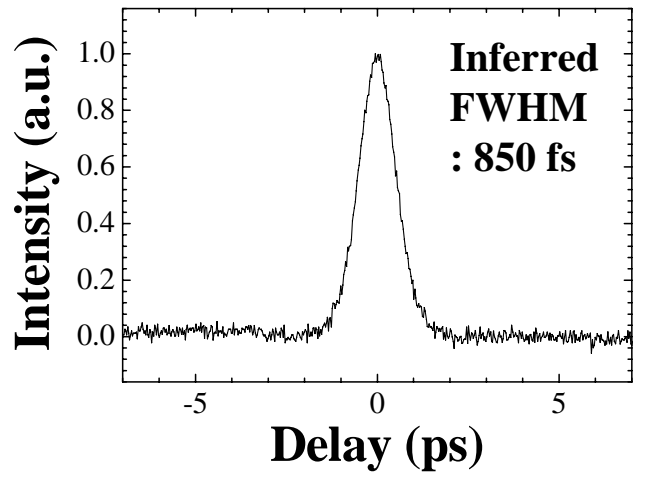

(b)

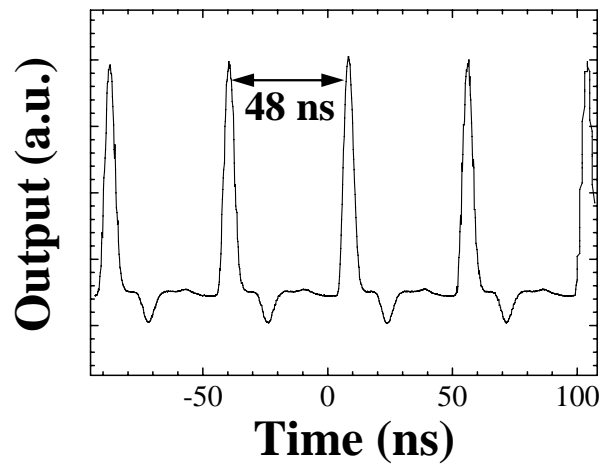

(c) 